\title{
Cortical maturation in fetuses referred for 'isolated' mild ventriculomegaly: a longitudinal ultrasound assessment
}

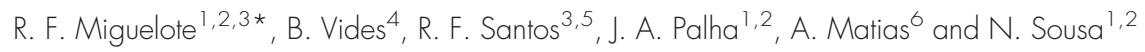 \\ 'Life and Health Sciences Research Institute (ICVS), School of Health Sciences, University of Minho, Braga, Portugal \\ ${ }^{2}$ CCVS/3Bs - PT Government Associate Laboratory, Braga/Guimarães, Portugal \\ ${ }^{3}$ Department of Obstetrics and Gynaecology, Centro Hospitalar do Alto Ave, Guimarães, Portugal \\ ${ }^{4}$ Department of Obstetrics and Gynaecology, Hospital de Braga, Braga, Portugal \\ ${ }^{5}$ Center for Research in Health Technologies and Information Systems (CINTESIS), Faculty of Medicine, University of Porto, Porto, Portugal \\ ${ }^{6}$ Department of Obstetrics and Gynaecology, Faculty of Medicine, Hospital of S. João, Porto, Portugal \\ *Correspondence to: Rui Filipe Miguelote. E-mail: rui.miguelote@gmail.com
}

\section{ABSTRACT}

Objectives To compare cortical maturation between fetuses with isolated mild ventriculomegaly (IMV) and healthy fetuses, and to explore its potential prognostic value in IMV.

Methods This prospective study quantified cortical maturation by ultrasound in 24 fetuses with IMV and 46 healthy fetuses. Depth and grading the developmental pattern of the parieto-occipital fissure (POF), calcarine fissure (CF) and sylvian fissure, and grading the Sylvian fissure operculization at 23-25, 27-28 and 31-32 weeks gestation were determined.

Results At 23-25 and 27-28 gestational weeks, POF and CF mean depths were statistically lower in the IMV group. The $\mathrm{POF}$ and CF depth distribution had a normal distribution in the control group but displayed a bimodal distribution in the IMV group. IMV with progression of ventricular dilatation showed mean depth of CF lower than IMV in which ventriculomegaly regressed or remained stable. The sensitivity, specificity, positive predictive value and negative predictive value of a CF depth below the fifth percentile to predict progression of ventricular dilatation were, at 28 weeks, $100 \%, 88 \%, 67 \%$ and $100 \%$, respectively.

Conclusions Cortical fissure assessment by ultrasound allowed the differentiation of a subgroup of IMV fetuses with a higher risk of progression of ventricular dilatation. (c) 2012 John Wiley \& Sons, Ltd.

\section{INTRODUCTION}

'Isolated' mild ventriculomegaly (IMV) poses a challenge for diagnosis and prenatal counseling. IMV or 'apparently' isolated ventriculomegaly is defined as a mild ventriculomegaly (ventricular atrial width of $10.0-15.0 \mathrm{~mm}$ ) without associated cerebral malformations or markers of fetal aneuploidy upon initial sonographic evaluation. ${ }^{1-3}$ IMV is, by definition, a provisional diagnosis in which the subsequent prenatal assessment may reveal cerebral or extracerebral malformations, congenital infections or chromosomal abnormalities. The rate of undetected associated malformations at the first examination varies considerably from $8 \%{ }^{4}$ to $40 \%{ }^{3}$ Although the prognosis of IMV is mainly dependent on the presence of associated cerebral malformations, it is also known that infants with confirmed IMV at birth have a higher rate of neurodevelopmental delay than that estimated for the general population $\left(10-15 \%^{1-3,5,6}\right.$ vs $\left.1-3 \%^{7}\right)$.
Most cases of ventricle dilatation correspond to a constitutive variant of normal; yet, in others, there may be an altered cerebral architecture that results in functional impairment. The malformations of cortical development (MCD) underlying these architectural changes may, however, be undetectable on prenatal ultrasound or magnetic resonance (MRI), which explains why many fetuses escape prenatal diagnosis of MCD. ${ }^{8}$ In fact, migration defects have diverse sonographic appearances that are usually not visible until the third trimester of pregnancy, unless subtle brain landmarks are sought. ${ }^{9}$

Cortical maturation has been assessed in fetuses with ventriculomegaly in two previous studies. ${ }^{10,11}$ However, the results of these studies, with regard to fetuses with IMV, were contradictory: Whereas Levine et al. ${ }^{10}$ found a 2-week mean lag in the time of appearance of the fissures or sulci in the IMV groups when comparing with healthy fetuses, Li et al. ${ }^{11}$ failed to find a statistically significant lag in sulcal visualization in fetuses 
with isolated ventriculomegaly compared with fetuses with normal cerebral findings. Thus, and taking into account recent sonographic methodological progresses for assessing cortical development, ${ }^{9,12-14}$ we thought of interest to explore the potential of cortical development ultrasound assessment for differentiating the cases of IMV.

\section{METHODS}

The present study is a prospective observational study involving a cohort of 24 fetuses referred because of IMV (cases) and a cohort of 46 healthy fetuses (controls). The study was approved by the local Ethics Committee, and all the pregnant women gave informed consent.

\section{Subjects}

IMV cases were referred to our study after an ultrasound examination, at second or third trimester, demonstrating IMV. IMV was defined as a dilation of the ventricular atrium between 10.0 and $15.0 \mathrm{~mm}$ without associated cerebral anomalies, soft markers of aneuploidy or structural malformation evident on ultrasound scan. Morphological ultrasound examination and confirmation of ventricular dilation were performed during initial ultrasound evaluation. The ventricular atrium was measured as proposed by the International Society of Ultrasound in Obstetrics and Gynecology guidelines. ${ }^{15}$ Controls were recruited from low-risk singleton pregnancies with a normal second-trimester fetal anatomic survey.

All cases and controls had the gestational age determined by a first-trimester sonogram and had negative screening for congenital infections (toxoplasmosis, rubella and cytomegalovirus). Karyotyping was offered to all cases. The cases and controls in which karyotyping was not performed were included in the study only after clinical exclusion of syndromes of chromosomal origin at birth. The anomalies commonly associated with ventriculomegaly (corpus callosum agenesis, Dandy-Walker malformation, Chiari malformation and encephalocele) were specifically investigated by a detailed examination of the central nervous system by sonography and, in the majority of the cases (18/24), also by MRI.

\section{Sonography}

Ultrasound follow-up was performed immediately after referral and, according to the gestational age of referral, at 23 25, 2728 and 3132 weeks of gestation. These ultrasound evaluations were used to assess the course of the ventriculomegaly, to exclude late-emerging morphological anomalies and to acquire 3D ultrasound volumes of the fetal brain for later offline analysis of the cortical maturation.

Ultrasound evaluation was performed transabdominally and transvaginally by a single operator (R.F.M.) using a Voluson 730 Expert ultrasound machine (GE Medical Systems, Kretz Ultrasound, Zipf, Austria) equipped with a $48 \mathrm{MHz}$ abdominal transducer and a $59 \mathrm{MHz}$ endocavity transducer.

Fetal brain 3D ultrasound volumes were acquired in transventricular, transthalamic and transcerebellar axial planes, as described by the International Society of Ultrasound in Obstetrics and Gynecology, ${ }^{15}$ with a sweep angle set at $45^{\circ}$ for fetuses at 24 weeks gestation and $60^{\circ}$ for fetuses at 32 weeks gestation. Acquisition quality was set at High 1. Depending on the fetal position, mid-coronal, transcerebellar coronal and sagittal planes were attempted by transabdominal and transvaginal ultrasound, and 3D volumes were acquired.

\section{Assessment of cortical maturation by offline analysis}

Offline analysis and measurement of the stored 3D volume data were performed using 4D View software version 9.1 (GE Medical Systems, Kretz Ultrasound, Zipf, Austria).

Fetal brain volumes were analyzed by multiplanar manipulations as previously described. ${ }^{16-18}$ A systematic approach was used in which the first step was to put the reference dot on the midline echo in the acquisition plane (box A) and align the midline echo with the $X$-axis. The same step was carried out in box B. These steps allow the obtainment of an accurate axial plane in box A, an accurate coronal plane in box $B$ and an accurate sagittal plane in box $C$. The volumes were manipulated to obtain sequentially (1) the transventricular plane, ${ }^{15}$ (2) the parallel cephalad plane in which the full POF depth was visualized, (3) the axial plane described by Quarello et $a .^{13}$ and (4) the transcerebellar coronal plane (box B) perpendicular to the transcerebellar axial plane (box A).

In the transventricular plane, the biparietal diameter, head circumference, the ventricular atrium, the depth of the insula and the depth of the SF were measured, and the SF was graded.

The POF was measured in the cephalic plane to the transventricular plane and subsequently graded. In the axial plane described by Quarello, the SFO was graded using the scoring sheet proposed by the same author. ${ }^{13}$ The depth of the $\mathrm{CF}$ was measured in the coronal transcerebellar plane perpendicular to the transcerebellar axial planes, and the CF was subsequently graded. The depth of the fissures was measured according to the methods proposed by Alonso et $a .^{9}{ }^{9}$ The cortical grading of fissures was performed using the scores developed by Pistorius et al. ${ }^{14}$

For standardization purposes, all measurements in the controls were performed in the brain hemisphere that was more distant from the probe, regardless of the fetal side. In the subject cases, the measurements of the POF and CF were performed in both sides; for the statistical analysis, the measurement used was the one obtained in the brain hemisphere more distal to the probe in cases of bilateral symmetric ventriculomegaly (same methodology used in controls), the more dilated side in cases of bilateral asymmetric ventriculomegaly (three cases) and the measurement of the dilated side in cases of unilateral ventriculomegaly.

\section{Pregnancy outcome and neonatal assessment}

Data on pregnancy outcome (type of delivery, and perinatal and neonatal morbidity and mortality) were available for all cases and controls. Neonatal examination was performed in all controls and cases in the first and second day after delivery. This examination consisted in a systematic 'head-to-toes' physical examination and in neurologic examination based in the Dubowitz neonatal neurological examination. ${ }^{19}$ This neurological examination included the assessment of tone, tone patterns, reflexes, movements, abnormal signs and some aspects of behavior. ${ }^{19}$ 


\section{Statistical analysis}

Summary statistics for continuous variables were depicted by using the mean and standard deviation, if its distribution was normal; otherwise, the median and centiles were utilized. Differences in categorical variables were analyzed by Fisher's Exact Test. Comparison of means was performed by twosample $t$-test, with a correction for unequal variances (Welch test) whenever the $p$-value of the $F$-test for equal variances was $<0.05$. Data not normally distributed were compared by Mann-Whitney $U$ test.

Regression analysis was used to test for relationship between the depth of the insula, SF, POF and CF, and the size of the lateral ventricles, for the combined sample (case and control subjects).

Analysis of IMV subgroups, categorized according to the course of ventriculomegaly, was performed. Logistic regression analysis was used to correlate CF and POF depth and the course of ventriculomegaly after adjusting for ventricular size.

The sensitivity, specificity, positive predictive value and negative predictive value of the $\mathrm{CF}$ and POF depth below the fifth percentile to predict progression of ventricular dilatation were calculated. The $95 \%$ confidence intervals for these proportions were calculated according to the efficient-score method (corrected for continuity) described by Newcombe and Robert. ${ }^{20} \mathrm{~A}$ significance level $(\alpha)$ of 0.05 , and two-sided tests, was considered.

The data were analyzed using MED-CALC version 12.1.0.0 (Medcalc Software, Mariakerke, Belgium) and Excel 2007 for Windows Vista (Microsoft Corp., Redmond, WA, USA).

\section{RESULTS}

Between January 2010 and December 2011, 29 fetuses with apparent IMV were referred to this study. Five cases were excluded: three cases in which ventricular dilatation was not confirmed, one case with congenital cytomegalovirus infection and one case with Dandy-Walker malformation. Ten cases (42\%) were referred at 21-25 weeks gestation, 11 (46\%) at 26-28 weeks and $3(13 \%)$ at 29-32 weeks gestation. The mean gestational age at diagnosis of IMV was 25.7 (range, 21-31) weeks. A total of 54 ultrasound examinations were performed in the IMV group: 10, 21 and 23 examinations at 2325, 2728 and 3132 weeks of gestation, respectively. One pregnant woman from the IMV group quit the study between the second and third ultrasound examinations. The digital recordings of two ultrasound examinations performed at 31-32 weeks gestation in two IMV fetuses were lost because of backup problems, and the cortical development assessment of these examinations was not performed.

The control group consisted of 46 healthy fetuses. A total of 130 ultrasound examinations were performed: 46, 45 and 39 examinations at 23-25, 27-28 and 31-32 weeks gestation, respectively. The control group had seven dropouts during the ultrasound follow-up.

The demographic characteristics of the two groups and the cerebral anthropometrics variables are presented in Tables 1 and 2, respectively.

Ventriculomegaly was unilateral in 10 cases $(41.7 \%)$, bilateral and symmetrical in 11 cases $(45.8 \%)$ and bilateral and
Table 1 Demographic characteristics

\begin{tabular}{lccr} 
& Control & IMV & $p$-value \\
\hline Maternal age (years) & $30.0 \pm 4.55$ & $30.5 \pm 5.27$ & 0.658 \\
\hline Fetus gender & & & \\
\hline Male & 22 & 15 & 0.315 \\
\hline Female & 24 & 9 & \\
\hline $\begin{array}{l}\text { Birth weight (kg) } \\
\text { Gestational age at }\end{array}$ & $3.297 \pm .378$ & $3.343 \pm .463$ & 0.656 \\
\hline birth (weeks) & $38.7 \pm 1.3$ & $38.8 \pm 1.0$ & 0.743 \\
\hline Apgar score at & & & \\
\hline 1 min & $8.93 \pm 0.75$ & $8.96 \pm 0.98$ & 0.887 \\
\hline 5 min & $9.89 \pm 0.32$ & $9.74 \pm 0.54$ & 0.149 \\
\hline
\end{tabular}

Values of mean $\pm S D$; IMV, isolated mild ventriculomegaly.

Table 2 Cerebral anthropometrics parameters for the fetuses with isolated mild ventriculomegaly and controls

\begin{tabular}{|c|c|c|c|}
\hline & Control & IMV & $p$-value \\
\hline \multicolumn{4}{|c|}{ Biparietal diameter $(\mathrm{mm})$} \\
\hline $23-25 \mathrm{~W}$ & $57.9 \pm 3.0$ & $59.8 \pm 2.2$ & 0.064 \\
\hline $27-28 W$ & $71.5 \pm 3.8$ & $73.5 \pm 4.6$ & 0.065 \\
\hline $31-32 W$ & $80.6 \pm 3.2$ & $82.3 \pm 3.7$ & 0.065 \\
\hline \multicolumn{4}{|c|}{ Head circumference $(\mathrm{mm})$} \\
\hline $23-25 \mathrm{~W}$ & $216 \pm 9.3$ & $225 \pm 5.5$ & 0.004 \\
\hline $27-28 \mathrm{~W}$ & $265 \pm 10.1$ & $273 \pm 13.7$ & 0.005 \\
\hline $31-32 W$ & $295 \pm 9.5$ & $303 \pm 14.5$ & 0.028 \\
\hline \multicolumn{4}{|c|}{$\begin{array}{l}\text { Lateral ventricle } \\
\text { width (mm) }\end{array}$} \\
\hline $23-25 \mathrm{~W}$ & $6.3 \pm 1.0$ & $10.9 \pm 0.7$ & $<0.001$ \\
\hline $27-28 \mathrm{~W}$ & $5.5 \pm 1.4$ & $10.8 \pm 1.1$ & $<0.001$ \\
\hline $31-32 W$ & $5.1 \pm 1.4$ & $10.0 \pm 1.7$ & $<0.001$ \\
\hline \multicolumn{4}{|c|}{$\begin{array}{l}\text { Corpus callosum } \\
\text { length (mm) }\end{array}$} \\
\hline $23-25 \mathrm{~W}$ & $27.4 \pm 2.0$ & $28.6 \pm 2.7$ & 0.125 \\
\hline $27-28 \mathrm{~W}$ & $34.7 \pm 1.2$ & $36.1 \pm 2.3$ & 0.017 \\
\hline $31-32 W$ & $38.0 \pm 1.7$ & $39.2 \pm 2.2$ & 0.018 \\
\hline \multicolumn{4}{|c|}{ Cerebellar vermis CC (mm) } \\
\hline $23-25 W$ & $13.4 \pm 0.6$ & $13.8 \pm 0.7$ & 0.072 \\
\hline $27-28 W$ & $17.3 \pm 1.3$ & $17.6 \pm 1.8$ & 0.378 \\
\hline $31-32 W$ & $19.7 \pm 1.1$ & $20.1 \pm 1.3$ & 0.210 \\
\hline
\end{tabular}

Values of mean $\pm S D ; I M V$, isolated mild ventriculomegaly; $W$, weeks; CC, craniocaudal diameter. Values in bold are variables with statistical significance $p<0.05$.

asymmetrical in 3 cases (12.5\%) (Table 3). The ventriculomegaly was located on the left side in eight (80\%) cases of unilateral ventriculomegaly. At diagnosis, the range of ventricular dimensions was 10.1 to $11.8 \mathrm{~mm}$ (mean $\pm \mathrm{SD}$ : $10.8 \pm 0.49$ ). During the antenatal ultrasound follow-up, ventriculomegaly resolved in $9(37.5 \%)$ cases, remained stable in $11(45.8 \%)$ and 
Table 3 Clinical data of 24 cases of isolated mild ventriculomegaly

\begin{tabular}{|c|c|c|c|c|c|c|c|c|}
\hline \multirow[b]{2}{*}{ Case } & \multirow{2}{*}{$\begin{array}{l}\text { Gestational age } \\
\text { at diagnosis } \\
\text { (weeks) }\end{array}$} & \multirow[b]{2}{*}{ Sex } & \multicolumn{2}{|c|}{ Atrium initial scan $(\mathrm{mm})$} & \multirow[b]{2}{*}{ Symmetry } & \multicolumn{2}{|c|}{ Atrium final scan $(\mathrm{mm})$} & \multirow{2}{*}{$\begin{array}{c}\text { Course of } \\
\text { ventriculomegaly }\end{array}$} \\
\hline & & & Left & Right & & Leff & Right & \\
\hline 1 & 31 & $\mathrm{~F}$ & 10.9 & 7.8 & Unilateral & 10.9 & 7.8 & Stable \\
\hline 2 & 23 & M & 11.3 & 6.8 & Unilateral & 11.2 & 7 & Stable \\
\hline 3 & 22 & $\mathrm{~F}$ & 11.7 & 10.1 & Bilateral & 9.6 & 8.4 & Resolved 32w \\
\hline 4 & 21 & M & 10.6 & 10.3 & Bilateral & 7.4 & 7.1 & Resolved 28w \\
\hline 5 & 21 & M & 11.8 & 8.6 & Unilateral & 12.1 & 7.6 & Stable \\
\hline 6 & 27 & M & 11.4 & 11.2 & Bilateral & 9.8 & 9.5 & Resolved 32w \\
\hline 7 & 28 & M & 11 & 10.8 & Bilateral & 10.8 & 10.1 & Stable \\
\hline 8 & 25 & M & 10.2 & 10.3 & Bilateral & 7.4 & 7.2 & Resolved 28w \\
\hline 9 & 26 & $\mathrm{~F}$ & 10.6 & 7.3 & Unilateral & 6.9 & 5 & Resolved 32 w \\
\hline 10 & 22 & M & 10.1 & 9.8 & Unilateral & 8.2 & 7.5 & Resolved 32w \\
\hline 11 & 26 & $\mathrm{~F}$ & 11.3 & 10.8 & Bilateral & 9.8 & 9.2 & Resolved 32w \\
\hline 12 & 27 & M & 10.5 & 10.8 & Bilateral & 9.2 & 8.8 & Resolved 32w \\
\hline 13 & 30 & $\mathrm{~F}$ & 10.9 & 10.3 & Bilateral & 10.8 & 10.2 & Stable \\
\hline 14 & 28 & M & 9.9 & 10.2 & Bilateral & 10.3 & 10.1 & Stable \\
\hline 15 & 27 & M & 10.2 & 7.3 & Unilateral & 12.9 & 7.6 & Progressed \\
\hline 16 & 22 & $\mathrm{~F}$ & 10.7 & 10.5 & Bilateral & 11.7 & 11.3 & Progressed \\
\hline 17 & 23 & M & 10.1 & 10 & Bilateral & 10.4 & 10.2 & Stable \\
\hline 18 & 27 & M & 10.8 & 8.7 & Unilateral & 10.8 & 8.2 & Stable \\
\hline 19 & 22 & M & 7.4 & 10.6 & Unilateral & 6.2 & 12.3 & Progressed \\
\hline 20 & 26 & M & 10.8 & 10.1 & Bilateral & 10.9 & 10.3 & Stable \\
\hline 21 & 28 & $\mathrm{~F}$ & 10.1 & 7.3 & Unilateral & 7.6 & 6.3 & Resolved 32w \\
\hline 22 & 31 & $\mathrm{~F}$ & 10.2 & 10.1 & Bilateral & 10.2 & 10.1 & Stable \\
\hline 23 & 27 & $\mathrm{~F}$ & 6.7 & 11 & Unilateral & 6.6 & 11.1 & Stable \\
\hline 24 & 21 & M & 10 & 10.7 & Bilateral & 12.6 & 13.7 & Progressed \\
\hline
\end{tabular}

$M$, male; F, female; w, weeks.

progressed (an increase of more than $3 \mathrm{~mm}$ ) in 4 (16.7\%; Table 3 ). The resolution occurred between 25 and 28 weeks in two cases and between 28 and 32 weeks in the remaining seven cases. Three of the four cases with progression of ventriculomegaly presented at 32 weeks gestation an atrium ventricular width of more than $12 \mathrm{~mm}$ (Table 3 ).

Associated extracerebral anomalies were found, during ultrasound follow-up or in the neonatal examination, in six (25\%) fetuses in the IMV group (Table 4). These abnormalities included perimembranous ventricular septal defect, pulmonary stenosis, situs inversus totalis, unilateral pyelectasia, agenesis of the nail of the left hand forefinger and two cases of dysmorphic facies (prominent forehead, elongated facies and low set ears; low set ears, hypertelorism and microphthalmia). In the control group, unilateral pyelectasia was found in two fetuses.

\section{MRI assessment}

MRI was performed in 18 (75\%) cases of IMV at a median gestational age of 32 weeks (range, 25-36). Ventriculomegaly was found in 16 cases. In the other two cases, MRI was performed when the ventriculomegaly had resolved. No other abnormalities were found on MRI.
Pregnancy outcome and neonatal assessment

The gestational ages at birth, Apgar scores and birth weights are shown in Table 1.

There were no cases of fetal or neonatal demise in both groups.

A neonate in the IMV group showed marked hypotonia, periods of apnea and bradypnea, a prominent forehead, elongated facies and low set ears. This neonate corresponds to a female fetus with bilateral ventriculomegaly (left atrium $10.8 \mathrm{~mm}$, right atrium $10.2 \mathrm{~mm}$ ) diagnosed at 30 weeks gestation (in the ultrasound examinations at 22 and 25 weeks, the atriums were below $10 \mathrm{~mm}$ ). At 2 months of life, this child maintained marked hypotonia, requiring nasogastric tube feeding, and showed a clear developmental delay. Screening for metabolic diseases was negative.

There were no other abnormal findings in the neurological examination of the neonates in both groups, and there were no cases of neonatal convulsions.

\section{Cortical maturation assessment}

The mean depth of POF was statistically significantly lower in the IMV group at 23-25 $(p=0.018)$ and $27-28$ weeks $(p=0.049)$ (Figure 1). The distribution of the POF depth in the control group 
Table 4 Gestational age at ventriculomegaly diagnosis, natural history and calcarine fissure depth of isolated mild ventriculomegaly cases with associated extracerebral anomalies

\begin{tabular}{|c|c|c|c|c|c|}
\hline $\begin{array}{l}\text { IMV cases with associated } \\
\text { extracerebral anomalies }\end{array}$ & $\begin{array}{c}\text { Gestational age at } \\
\text { ventriculomegaly diagnosis } \\
\text { (weeks) }\end{array}$ & $\begin{array}{l}\text { Course of } \\
\text { VM }\end{array}$ & $\begin{array}{l}\text { CF depth at } \\
24 \mathrm{w}(\mathrm{mm})\end{array}$ & $\begin{array}{l}\text { CF depth at } \\
28 \mathrm{w}(\mathrm{mm})\end{array}$ & $\begin{array}{l}\text { CF depth at } \\
32 \mathrm{w}(\mathrm{mm})\end{array}$ \\
\hline Case 6 - Unilateral pyelectasia & $27 w$ & Resolved & - & $<\mathrm{P} 5$ & $<$ P5 \\
\hline $\begin{array}{l}\text { Case } 11 \text { - Perimembranous ventricular } \\
\text { septal defect }\end{array}$ & $26 w$ & Resolved & - & P25-50 & P25-50 \\
\hline Case 12 - Agenesis of the nail of the forefinger & $27 w$ & Resolved & - & P25-50 & P10-25 \\
\hline $\begin{array}{l}\text { Case } 13 \text { - Dysmorphic facies and marked } \\
\text { hypotonia in the neonatal period }\end{array}$ & $30 w$ & Persisted & - & - & P5-10 \\
\hline $\begin{array}{l}\text { Case } 16 \text { - Dysmorphic facies and } \\
\text { pulmonary stenosis }\end{array}$ & $22 w$ & Progressed & $<P 5$ & $<$ P5 & (a) \\
\hline Case 17 - Situs inversus totalis & $23 w$ & Persisted & $<P 5$ & $<\mathrm{P} 5$ & $<\mathrm{P} 5$ \\
\hline
\end{tabular}

IMV, isolated mild ventriculomegaly; VM, ventriculomegaly; CF, calcarine fissure; w, weeks.

${ }^{a}$ Cortical maturation assessment not performed because of loss of ultrasound examination.
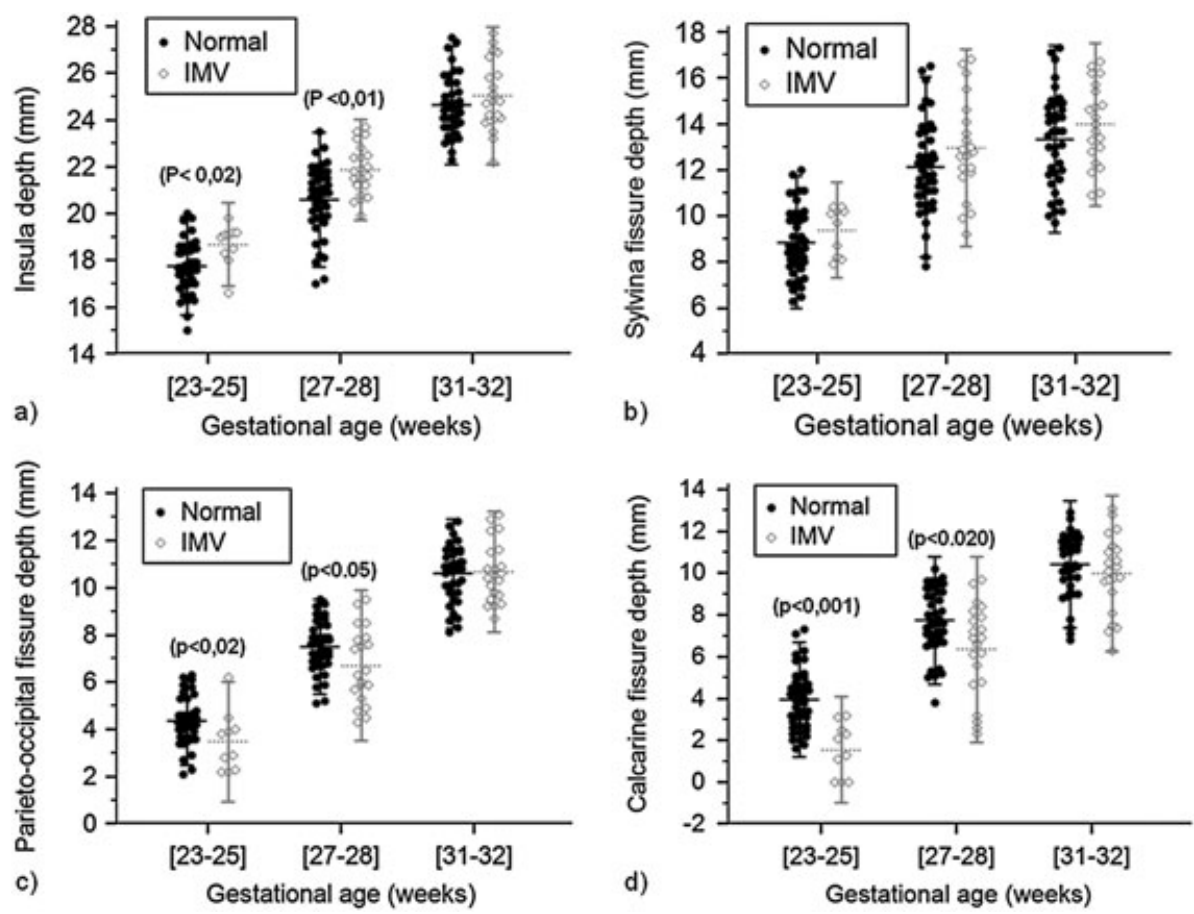

Figure 1 (a) Depth of the insula, (b) sylvian fissure, (c) parieto-occipital fissure and (d) calcarine fissure in normal fetuses and in fetuses with isolated ventriculomegaly (IMV) between 23 and 32 weeks gestation. The means and two standard deviations are shown

was close to the normal distribution, whereas in the IMV group, it was closest to bimodal distribution. The POF depth was below the fifth percentile of the control group in 30\% (95\%CI 8.1-64.6) of the fetuses with IMV at 23-25 weeks gestation and in $23.8 \%$ (95\%CI 9.1-47.6) at 27-28 weeks gestation. Similarly, the mean depth of the CF was statistically significantly lower in the IMV group for the gestational ages of 23-25 $(p<0.001)$ and $27-28$ weeks $(p=0.020$ ) (Figure 1). The CF depth was below the fifth percentile of the control group in $50 \%$ (95\%CI 20.1-79.9) of fetuses with IMV at 23-25 weeks gestation and in $28.6 \%$ (95\% CI 12.1-52.3) at 27-28 weeks gestation. In unilateral ventriculomegaly cases, the CF and POF depths were smaller on the dilated side (Figure 2). In four of these cases $(44.4 \%)$, this difference was marked (the fissure depth on the dilated side measured less than half of the fissure depth on the nondilated side).

A statistically significant inverse relationship between the depth of CF and the size of the lateral ventricle was noted for the total sample (case and control subjects), at the gestational ages of 23-25 and 27-28 weeks gestation (CF depth in $\mathrm{mm}$ at $23-25$ weeks gestation $=6.38+(-0.40 \times$ ventricular size); $r^{2}=0.24, \quad p<0.001$; CF depth in $\mathrm{mm}$ at $27-28$ weeks gestation $=9.24+(-0.27 \times$ ventricular size $\left.) ; r^{2}=0.16, p<0.001\right)$ (Figure 3). We did not find a statistically significant relationship between the depth of insula, sylvian fissure or POF, and the size of the lateral ventricle. There was no significant difference 


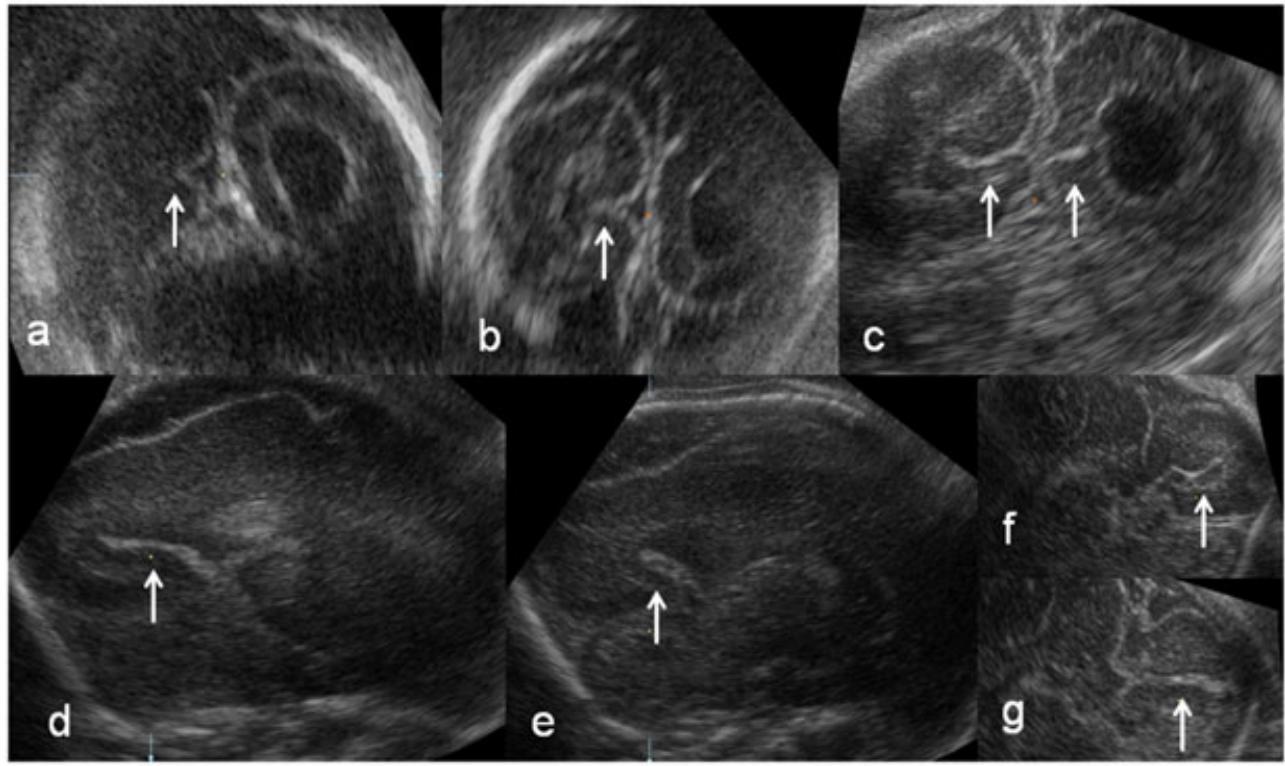

Figure 2 Asymmetry in the development of calcarine fissure (arrows) in a fetus with unilateral mild ventriculomegaly. Transcerebellar coronal ultrasound image at (a) 24 weeks, (b) 27 weeks and (c) 31 weeks. Parasagittal ultrasound image of the affected side (d) and non-affected sides (e) at 27 weeks and at 31 weeks $(f)$ and $(g)$, respectively
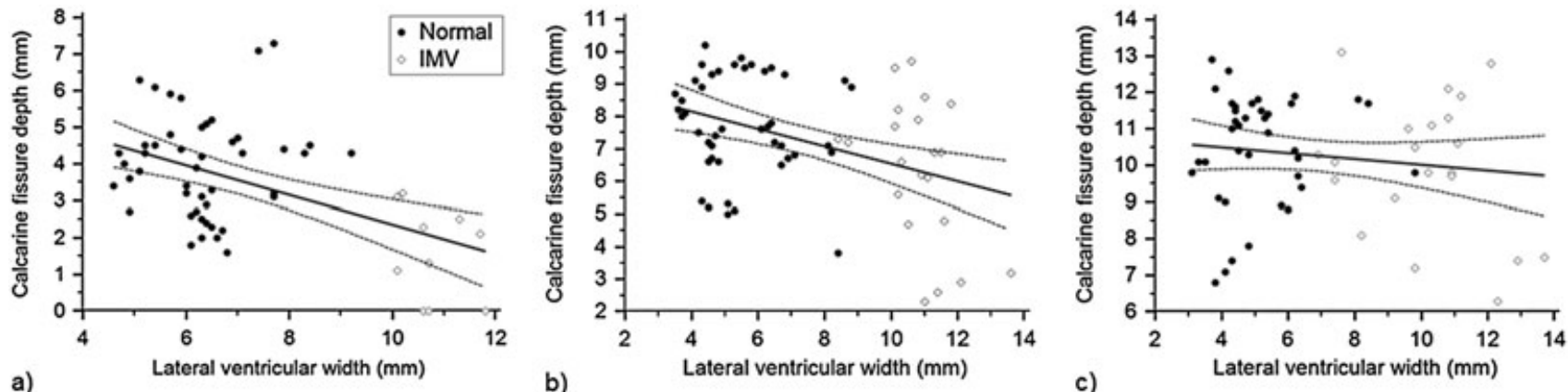

Figure 3 Relationship between the size of the lateral ventricles and calcarine fissure depth, at (a) 23-25 weeks, (b) 27-28 weeks and (c) 31-32 weeks. The regression line and 95\% confidence interval are shown. IMV, isolated ventriculomegaly

in the mean depth of the $\mathrm{CF}$, POF, insula, and sylvian fissure between female and male fetuses in either group (controls and IMV). In the analysis of cortical maturation by subgroup according to the progression of ventricular dilatation, the subgroup of IMV with progression of ventriculomegaly showed a mean depth of CF lower than the control group and than the other subgroups of IMV in which the ventriculomegaly regressed or stabilized (Table 5). The difference between the control group and the subgroup of IMV with progression was statistically significant at all gestational ages (Table 5). The

Table 5 The progression of the calcarine fissure depth during the gestation in the control group and in the subgroups of ventriculomegaly grouped by the course of ventriculomegaly

\begin{tabular}{|c|c|c|c|}
\hline & $23-25 w$ & $27-28 w$ & $31-32 w$ \\
\hline Control & $3.95 \pm 1.37(1.6-7.3)$ & $7.74 \pm 1.53(3.8-10.2)$ & $10.56 \pm 1.31(6.8-12.9)$ \\
\hline Resolved & $2.68 \pm 0.57(2.1-3.2)$ & $6.7 \pm 1.87(2.6-9.7)$ & $10.64 \pm 1.58(7.3-12.8)$ \\
\hline Persisted & $1.20 \pm 1.25(0-2.5)$ & $7.0 \pm 2.48(2.3-9.5)$ & $9.89 \pm 1.71(7.2-13.1)$ \\
\hline Progressed & $0.43 \pm 0.75(0-1.3)^{*}$ & $3.83 \pm 0.92(2.9-4.8)^{*}$ & $6.90 \pm 0.85(6.3-7.5)^{*}$ \\
\hline
\end{tabular}

Values of mean $\pm \mathrm{SD}$ (range) in $\mathrm{mm}$; w, weeks.

* p-value $<0.05$ for the difference between control group and the subgroup. 
difference between the subgroup of IMV with progression and the subgroup in which the ventriculomegaly regressed or stabilized was near the statistical significance in the assessment at 23-25 weeks ( $p=0.058)$ and was statistically significant in the assessment at $27-28$ weeks ( $p=0.021$ ). CF depth was predictive of progression of ventriculomegaly at 23-25 weeks gestation $(p=0.037)$ and at $27-28$ weeks gestation $(p=0.021)$ as assessed by ordinal logistic regression after adjusting for ventricular size. The sensitivity, specificity, positive predictive value, negative predictive value and likelihood ratios of the CF depth below the fifth percentile to predict progression of ventricular dilatation are showed in Table 6.

The mean depth of POF was also lower in the subgroup of IMV with progression of ventricular dilatation; however, its difference and the prognostic value of POF depth below the fifth percentile to predict ventriculomegaly progression did not reach statistical significance at any gestational age.

The ventricular width at the time of diagnosis, the bilaterality and the sex of the fetus were not associated with the ventriculomegaly progression.

The analysis of cortical maturation by subgroup according to the presence of associated extracerebral malformations was not performed because of the small number of cases with malformations $(n=6)$, late ventriculomegaly onset/diagnosis (after 27weeks) in four of these cases and heterogeneity of malformations observed. Nevertheless, the analysis of cases showed that the case with dysmorphic syndrome and pulmonary stenosis, the case with situs inversus totalis and the case with unilateral pyelectasia had CF depth below the fifth percentile (Table 4). The case with dysmorphic facies and marked hypotonia in the neonatal period presented ventricular dilatation only at 30 weeks gestation $(8.3 \mathrm{~mm}$ in 22 weeks gestation examination) and so the cortical assessment was performed only at 31 weeks gestation; at this age, this fetus presented CF depth between the fifth and tenth percentiles of the control group (Table 4).

Cortical grading of CF, POF and sylvian fissure as assessed by the Pistorius method was not statistically different at any gestational age. The scoring of the sylvian fissure operculization was also not statistically different between these groups (Figure 4). However, one fetus in the IMV group showed a marked delay in SFO (Figures 4 and 5).

Morphological assessment of the fissures revealed an abnormal POF in one fetus with unilateral IMV (Figure 6). The abnormal POF was on the non-dilated side and was too deep and ran parallel to the $\mathrm{CF}$, instead of the normal upwards and

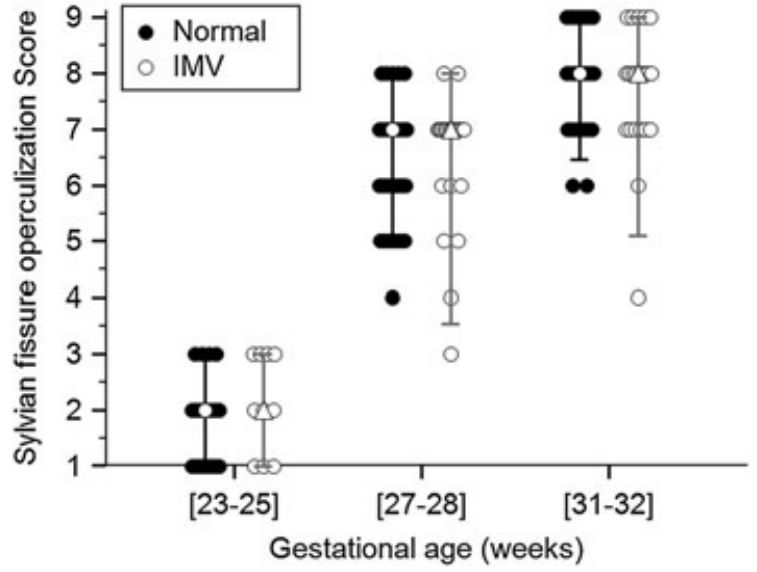

Figure 4 Grade of sylvian fissure operculization (SFO) assessed by the Quarello method in normal fetuses and in fetuses with isolated mid ventriculomegaly (IMV). The median and the 5th and 95th percentiles are shown

backwards route. The POF of the dilated side was too shallow for the gestational age.

No abnormal fissures were found in the control group.

\section{DISCUSSION}

This study provides the first description of differences in cortical maturation, identifiable by ultrasound, between fetuses with IMV and healthy fetuses. These differences were statistically significant for the depth of CF and were mainly observed in a subgroup of IMV that showed progression of ventricular dilation. CF depth below the fifth percentile proved to be a risk marker for progression of ventricular dilatation, which is, together with the presence of associated extracerebral malformations, the most important prognostic factors for abnormal postnatal development in IMV fetuses. Our study does not present a postnatal follow-up, but the association between CF depth below the fifth percentile and one of the most important prognostic factors for abnormal postnatal development in IMV fetuses strongly suggests that evaluation of cortical maturation at the initial evaluation of IMV cases may be a predictor of postnatal development. This hypothesis is also supported by a recent study ${ }^{11}$ of cortical maturation assessment by MR and sonography in fetuses with ventriculomegaly and with a postnatal follow-up of 3 years. In that study, the odds ratio of normal postnatal neurodevelopment

Table 6 Sensitivity, specificity, positive and negative predictive values, and odds ratio of calcarine fissure depth below the fifth percentile at 23-24 and 27-28 weeks gestation to predict progression of ventricular dilatation

\begin{tabular}{|c|c|c|c|c|c|c|c|c|}
\hline & \multirow{2}{*}{$\begin{array}{l}\text { Gestational } \\
\text { age (weeks) }\end{array}$} & \multicolumn{2}{|c|}{ Sensitivity } & \multicolumn{2}{|c|}{ Specificity } & \multirow{2}{*}{$\begin{array}{c}\text { PPV } \\
\%(95 \% \mathrm{Cl})\end{array}$} & \multirow{2}{*}{$\begin{array}{c}\text { NPV } \\
\%(95 \% \mathrm{Cl})\end{array}$} & \multirow{2}{*}{$\begin{array}{c}\text { OR } \\
(95 \% \mathrm{Cl})\end{array}$} \\
\hline & & $n$ & $\%(95 \% \mathrm{Cl})$ & $n$ & $\%(95 \% \mathrm{Cl})$ & & & \\
\hline \multirow[t]{2}{*}{ Progression } & 23-25 & $3 / 3$ & $100(31-100)$ & $5 / 7$ & $71.4(30-95)$ & 60 (17-93) & $100(46-100)$ & $3.5(1.1-1) .3)$ \\
\hline & $27-28$ & $4 / 4$ & $100(40-100)$ & $15 / 17$ & $88.2(62-98)$ & $67.7(24-94)$ & $100(75-100)$ & $8.5(2.3-31.2)$ \\
\hline
\end{tabular}

PPV, positive predictive value; NPV, negative predictive value; OR, odds ratio. 

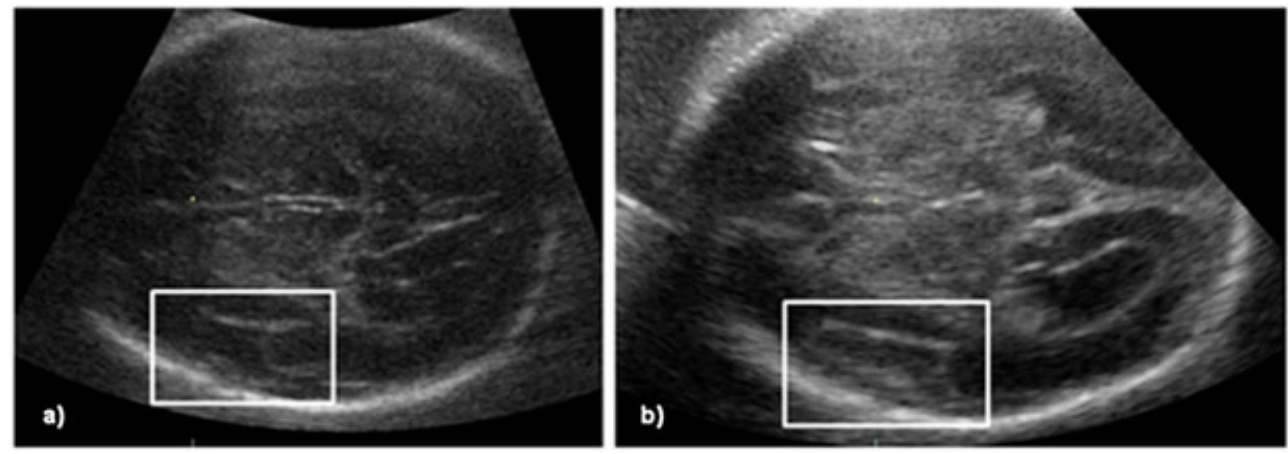

Figure 5 Grade of sylvian fissure operculization (SFO) in two fetuses with isolated mid ventriculomegaly. (a) Fetus at 27 weeks gestation with a normal grade of SFO (a score of 7 by the Quarello method); (b) fetus at 27 weeks and 2 days with a delay of SFO (a score of 3)

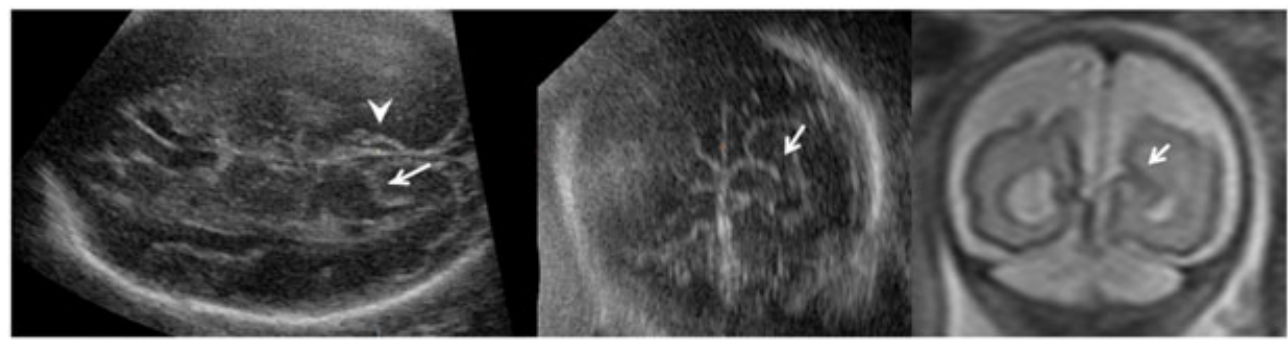

Figure 6 Abnormal orientation and depth of the parieto-occipital fissure (arrow) in the unaffected side and shallow parieto-occipital fissure (arrowhead) in the dilated side in a fetus with unilateral mild ventriculomegaly at 28 weeks and 4 days of gestation. (a) Axial US image, (b) transcerebellar coronal US image and (c) coronal magnetic resonance image at 29 weeks

of fetus with ventriculomegaly was higher when the CF or POF had been visualized, on MR images, relatively to when it had not been visualized. ${ }^{11}$ Importantly, in that study, ${ }^{11}$ the visualization of these fissures by ultrasound did not show statistically significant prognostic value; in contrast, herein a more detailed fissure maturation assessment, with the evaluation of fissure morphology and fissure depth, unraveled a statistically significant prognostic value of cortical assessment by ultrasound.

The pathophysiology behind the lower depth of these fissures in some fetuses with IMV is not obvious. The 'apparent' delay in fissure maturation may simply be a consequence of deformation caused by the ventricular dilation or may reflect changes in brain architecture arising from alterations in cerebral neuronal migration or proliferation. Given that shallow fissures were not, in our study, a finding common to all IMV cases, we suggest that the lower fissure depth cannot be exclusively explained by a direct effect of ventricular dilation.

The relationship between the cortical development and the ventricular size in healthy fetuses was first suggested by Chi et al., ${ }^{21}$ based on the observation that the volume of the lateral ventricle begins to decrease following the appearance of calcarine sulcus. The underlying basis for observation is provided by recent studies in monkey fetuse ${ }^{22}$ and in human fetuse $^{23}$ using MRI images: During the fetal period, the decrease in ventricular volume is accompanied by a decrease in the volume of the germinal matrix around the lateral ventricles and by an increase in the depth of the calcarine sulcus and an expansion of the subcortical structures. ${ }^{22,23}$
These suggest that, in some IMV fetuses, the delay in the migration of neurons from the germinal matrix, located on the lateral ventricular walls, to the cortex, may be the cause of both changes found in the brain architecture (ventricular dilatation and fissure maturation delay).

In support of this view, in a study using MRI images of 34 neonates with IMV on prenatal ultrasound, the prenatal enlargement of the lateral ventricle was associated with delayed or abnormal maturation of white matter in the neonatal period. ${ }^{24}$ In the context of cortical maturation abnormalities, the present observation of a marked delay in SFO in IMV is also of relevance given that SFO abnormalities have been increasingly diagnosed in infants and children with developmental delay. ${ }^{25}$ In fact, the development of the SF is one of the major brain maturational processes occurring in fetal life ${ }^{26}$ and can be used to depict either delayed maturation or pathological anomalies of the operculization process, which can reflect more diffuse gyral anomalies. ${ }^{27}$

A note of caution is also due here to highlight that in our study and in the aforementioned study, ${ }^{24}$ differences in cortical development were observed in fetuses presenting, at the initial evaluation, ventricular widths below $12 \mathrm{~mm}$. Therefore, the view that ventricular widths between 10 and $12 \mathrm{~mm}$ should be considered normal $^{28}$ should be carefully reappraised.

This study highlights the potential role that the fissure maturation assessment may have in prenatal counseling of IMV cases, as it may allow the distinction between cases in which the ventriculomegaly represent a constitutive variant of 
normal and cases in which the ventriculomegaly is the result of alterations in neuronal migration or proliferation. However, the clinical validation of these results requires its confirmation in studies with a higher number of cases and controls and, most importantly, with a longer postnatal follow-up for both groups. The importance of long-term follow-up in studies of IMV was highlighted by Patel et al., ${ }^{29}$ who found that $80 \%$ of the cognitive impairments detected during and up to 4 years of follow-up had not been detected at 1 year of age. Therefore, future studies on the same cohort will allow us to understand further how predictive are these measurements in terms of neurodevelopment impairment.

In conclusion, the maturation assessment of $\mathrm{CF}$ depth is proposed to be a predictor of progression of ventricular dilatation, and therefore, its assessment is likely to be of relevance in the prenatal counseling of IMV cases.

\section{WHAT'S ALREADY KNOWN ABOUT THIS TOPIC?}

- The most important prognostic factors in IMV are the association with other abnormalities not detected at first examination and the progression of ventricular dilatation; however, both of which are retrospective diagnoses.

- A recent study in cynomolgus monkey fetuses showed that the morphological maturation of the lateral ventricle is linked to cortical maturation

\section{WHAT DOES THIS STUDY ADD?}

- This study describes differences in cortical maturation, identifiable by ultrasound, between fetuses with IMV and healthy controls.

- Calcarine fissure depth below the fifth percentile predicts progression of ventricular dilatation in cases of 'apparently' IMV.

\section{REFERENCES}

1. Melchiorre K, Bhide A, Gika AD, et al. Counseling in isolated mild fetal ventriculomegaly. Ultrasound Obstet Gynecol 2009;34:212-24.

2. Ouahba J, Luton D, Vuillard E, et al. Prenatal isolated mild ventriculomegaly outcome in 167 cases. BJOG 2006;113:1072-9.

3. Kelly EN, Allen VM, Seaward G, et al. Mild ventriculomegaly in the fetus, natural history, associated findings and outcome of isolated mild ventriculomegaly: a literature review. Prenat Diagn 2001;21:697-700.

4. Pilu G, Falco P, Gabrielli S, et al. The clinical significance of fetal isolated cerebral borderline ventriculomegaly: report of 31 cases and review of the literature. Ultrasound Obstet Gynecol 1999;14:320-6.

5. Falip C, Blanc N, Maes E, et al. Postnatal clinical and imaging follow-up of infants with prenatal isolated mild ventriculomegaly a series of 101 cases. Pediatr Radiol 2007;37:981-9.

6. Laskin MD, Kingdom J, Toi A, et al. Perinatal and neurodevelopmental outcome with isolated fetal ventriculomegaly: a systematic review. J Matern Fetal Neonatal Med 2005;18:289-98.

7. Moeschler JB, Shevell M, American Academy of Pediatrics Committee on Genetics. Clinical genetic evaluation of the child with mental retardation or developmental delays. Pediatrics 2006;117:2304-16.

8. Malinger G, Lev D, Lerman-Sagie T. Abnormal sulcation as an early sign for migration disorders. Ultrasound Obstet Gynecol 2004;24:704-5.

9. Alonso I, Borenstein M, Grant G, et al. Depth of brain fissures in normal fetuses by prenatal ultrasound between 19 and 30 weeks of gestation. Ultrasound Obstet Gynecol 2010;36(6):693-9.

10. Levine D, Barnes PD. Cortical maturation in normal and abnormal fetuses as assessed with prenatal MR imaging. Radiology 1999;210:751-8.

11. Li Y, Estroff JA, Mehta TS, et al. Ultrasound and MRI of fetuses with ventriculomegaly: can cortical development be used to predict postnatal outcome? AJR Am J Roentgenol 2011;196:1457-67.

12. Mittal P, Gonçalves LF, Kusanovic JP, et al. Objective evaluation of sylvian fissure development by multiplanar 3-dimensional ultrasonography. J Ultrasound Med 2007;26:347-53.

13. Quarello E, Stirnemann J, Ville Y, Guibaud L. Assessment of fetal sylvian fissure operculization between 22 and 32 weeks a subjective approach. Ultrasound Obstet Gynecol 2008;32:44-9.

14. Pistorius LR, Stoutenbeek P, Groenendaal F, et al. Grade and symmetry of normal fetal cortical development: a longitudinal two- and threedimensional ultrasound study. Ultrasound Obstet Gynecol 2010;36:700-8.

15. International Society of Ultrasound in Obstetrics \& Gynecology Education Committee. Sonographic examination of the fetal central nervous system guidelines for performing the basic examination and the fetal neurosonogram. Ultrasound Obstet Gynecol 2007;29:109-16.

16. Bornstein E, Monteagudo A, Santos R, et al. A systematic technique using 3-dimensional ultrasound provides a simple and reproducible mode to evaluate the corpus callosum. Am J Obstet Gynecol 2010;202:201e1-5.

17. Pilu G, Ghi T, Carletti A, et al. Three-dimensional ultrasound examination of the fetal central nervous system. Ultrasound Obstet Gynecol 2007;30:233-45.

18. Bornstein E, Monteagudo A, Santos R, et al. Basic as well as detailed neurosonograms can be performed by offline analysis of threedimensional fetal brain volumes. Ultrasound Obstet Gynecol 2010;36:20-5.

19. Dubowitz LMS, Dubowitz V, Mercuri E. The Neurological Assessment of the Preterm and Full-term Infant (2nd edn). Mac Keith Press: London, 1999.

20. Newcombe RG. Two-sided confidence intervals for the single proportion: comparison of seven methods. Stat Med 1998;17:857-72.

21. Chi JG, Dooling EC, Gilles FH. Gyral development of the human brain. Ann Neurol 1977;1:86-93.

22. Fukunishi K, Sawada K, Kashima M, et al. Correlation between formation of the calcarine sulcus and morphological maturation of the lateral ventricle in cynomolgus monkey fetuses. Acta Neurobiol Exp 2011;71:381-6.

23. Kinoshita Y, Okudera T, Tsuru E, Yokota A. Volumetric analysis of the germinal matrix and lateral ventricles performed using MR images of postmortem fetuses. Am J Neuroradiol 2001;22:382-8.

24. Gilmore JH, Smith LC, Wolfe HM, et al. Prenatal mild ventriculomegaly predicts abnormal development of the neonatal brain. Biol Psychiatry 2008;64(12):1069-76.

25. Chen CY, Zimmerman RA, Faro S, et al. MR of the cerebral operculum: abnormal opercular formation in infants and children. AJNR Am J Neuroradiol 1996;17(7):1303-11.

26. Lerman-Sagie T, Malinger G. Focus on the fetal sylvian fissure. Ultrasound Obstet Gynecol 2008;32:3-4.

27. Guibaud L, Selleret L, Larroche JC, et al. Abnormal sylvian fissure on prenatal cerebral imaging: significance and correlation with neuropathological and postnatal data. Ultrasound Obstet Gynecol 2008;32(1):50-60.

28. Signorelli M, Tiberti A, Valseriati D, Molin E, et al. Width of the fetal lateral ventricular atrium between 10 and $12 \mathrm{~mm}$ : a simple variation of the norm? Ultrasound Obstet Gynecol 2004;23(1):14-8.

29. Patel MD, Filly AL, Hersh DR, Goldstein RB. Isolated mild fetal cerebral ventriculomegaly: clinical course and outcome. Radiology 1994;192:759-64. 